\title{
Psychological Stress Prediction on Social Media using Convolutional Neural Network
}

\author{
Preethi Rajam C.R., Uma Maheswari N., Jeyanthi S., Somasundaram S.K.
}

\begin{abstract}
Psychological stress which is a mental illness also causes physical problems to the human. Nowadays social media plays an important role in the world for communication to share their thoughts with their friends and family. The social media analysis is the process of detecting and predicting the user's thoughts and opinions which also one of the important perspective in the developing business environment. The overwhelming stress and long term stress sometimes lead to suicidal ideation. By analyzing the social media content to predict the overwhelming stress state of the users in the earlier stage will reduce the psychological stress and suicidal rate too. In this paper, we address the problem of stress prediction by using social media. The machine learning and deep learning methods to perform the classification of stress analysis. Here both image and text- tweet data are used and the images are processed with the Optical Character Recognition and the text data are processed by using the Natural Language Processing and Convolutional Neural Network for classifying the tweet content of the user as stressed or non-stressed. Furthermore, with the advancement of the machine learning and deep learning method of classification gives a better result in terms of performance and accuracy of the prediction.
\end{abstract}

Keywords: Social Media, Machine Learning, Natural Language Processing, Convolutional Neural Network.

\section{INTRODUCTION}

The social media like Facebook, Twitter, and WhatsApp are the popular social networking website where the users create and use these social media application for conveying the message and expressing their individual feelings, thoughts to their friends and family on different subjects. The social media applications are highly influencing people of all age group peoples nowadays and changing the lifestyle of the people. As the continuous use of social media by the users, it is much more possible to identify the psychological state of the user by gathering their social media message and communication data timely and by analyzing the content.

Psychological stress which is a medical and physical illness that is a threat to the people health. Year by year the stress level was increasing to the people and sometimes the overwhelming stress leads to suicidal ideation. In the year

Revised Version Manuscript Received on 10, September 2019.

Preethi RajamC.R., PG Scholar, Department of Computer Science and Engineering, PSNA College of Engineering and Technology, Dindigul, Tamilnadu, India.(Email: preethi96sai@gmail.com)

Uma Maheswari N., Professor, Department of Computer Science and Engineering, PSNA College of Engineering and Technology, Dindigul, Tamilnadu, India.(email: numamahi@gmail.com)

Jeyanthi S., Assistant Professor, Department of Computer Science and Tamilnadu, India.

Somasundaram S.K., Associate Professor, Department of Computer Science and Engineering, PSNA College of Engineering and Technology, Dindigul, Tamilnadu, India. Engineering, PSNA College of Engineering and Technology, Dindigul,

2018, about 15.7 per $100 \mathrm{k}$ people were suicide in India. Thought the stress became more common in our day to day life with being harmful to the human life. So there is much important to predict the stress level of the people before it turns into a severe problem. Some of the traditional methods to detect are stress are actually reactive and has some limitation like hysteretic, time and labor consuming and computationally expensive. As the increased use of social media application by all age group it much more possible to find the emotional or stress state of the user in the earlier stage by using machine learning techniques which is much better than the traditional methods.

\section{RELATED WORKS}

Machine learning is the trending technology used for "Decision making" or "Prediction making". It explores the construction of an algorithm which learns from data and predicts result by strictly following the conditions for higher accuracy. Supervised machine learning which consists of <input, output> pair and the main goal is to learn from the general rule to map the input and output. Here the data are trained to predict the desired result. Machine learning supervised learning approach for classification which plays a major role to predict the stress state of the user in the social network. There are many methods used for classification such as Naive Bayes, Maximum entropy, Random Forest, Decision tree classification, SVM, MLP classifier etc. By using the machine learning approaches is possible to identify the user behavior and stress level in social media.

The advancement of the social media application, there is more possible features are provided to the users to handle the application with the text, image and videos. To share the opinion the social media users widely using the images with text character. Recognition of characters from the image is not an easy task and it has the limitation of non-uniform lighting, low resolution, blur effect etc. 1 Mainly the text detection was done based on texture and region-based method. To extract the covariant region in the image in the blob detection, Maximally Stable Extremal Regions (MSRE) is used in. Its main process is to detect the region of text in the images and aggregate the region with similar intensity values at various thresholds. $2 \mathrm{~A}$ holistic word recognition framework consists of a gradient-based feature used for the images generated from lexicon words. This framework consists of Novel weighted Dynamic Time Warping (wDTW) approach that recognizes the text in the images by 


\section{PSYCHOLOGICAL STRESS PREDICTION ON SOCIAL MEDIA USING CONVOLUTIONAL NEURAL NETWORK}

matching the scene and synthetic image features. 3 The sliding window procedure to detect the character as a single visual word and the lexicon is used to group the characters to form a word with a limitation of maximum 500 words and it has the bigger impact on the performance. The character or text recognition using Optical Character Recognition (OCR) which gives higher accuracy when compared to all other recognition methods. It scans the text sequentially in the images to recognize the separate letters from the text line and it can be grouped.

Natural language processing (NLP) is a combination of computer science and artificial intelligence. It is the process and analyses large amounts of natural language data with the help of the computer program. NLP which consist of some basic process like Structure extraction, Identify and mark sentence, Language identification, Tokenization, Entity extraction and Phrase extraction, Lemmatization, and Stemming. 4 NLP with sentiment analysis that extracts the emotion combined with the polarity for a specific subject in the document. 5 The marginal framework is used in for the automatic identification of user's online behavioral changes to detect the suicide warning. The NLP is combined with the framework and detect the changes through behavioral and textual features. 6 Fuzzy ontology based sentiment analysis and Semantic Web Rule Language (SWRL) and rule-based decision making to monitor the transport activities. This system retrieves the tweets related to the transportation activities and features of the city and makes the decision by classifying the tweets. 7 Multinomial Naïve Bayes (MNB) and Multi-Layer Perceptron (MLP) method were used to identify the suicidal ideation prediction model based on Sina Microblog for the Chinese language. 8 Metropolis-Hasting algorithm is used for predicting the emotion in the social network. It predicts the emotion status of the people and stated that it is better than the several baseline methods. 9 Cross-validated machine learning models and using the programming codes consist the bags-of-word, tokens and unigram as features to detect the suicidality through twitter. 10 Deep Sparse Neural Network with cross-media microblog data is used for stress detection and uses the three-level framework to incorporate different features which are quite feasible. Due to the overwhelming stress, the depression and the suicide rate is increasing day by day. 11 To identify the depression level of the user in social media various methods had been done in the existing systems, in which the depression level of a person by observing and extracting emotions from the text, using emotion theories, machine learning techniques. The traditional classification algorithms like Support Vector Machine, Naive Bayes, and Maximum Entropy are used to classify the text contents. These algorithms are binary classifiers and probability classifiers and it has some limitation too.

In this paper to predict the psychological stress level in social media, Optical Character Recognition (OCR), Natural Language Processing (NLP) and Convolutional Neural Network (CNN) is used that mainly focused to improve the performance in terms of efficiency and accuracy.

\section{PSYCHOLOGICAL STRESS PREDICTION USING CNN \& RESULTS}

As the cardinal symptom of psychological stress is severe negative emotions and lack of positive emotions, which can be identified by using the machine learning approaches. The proposed method mainly consists of Optical Character Recognition for image processing, Natural Language Processing (NLP) and Convolutional Neural Network (CNN) for text content processing. The main components of the proposed system are Image processing that detects and extracts text-tweet from the images, Preprocessing, Feature extraction, Classification and Connecting to NGO as shown in Figure 1. The data was collected from social media like Twitter, Facebook etc. The image and text data set was used to predict the stress level of the user. The image dataset is collected and processed with OCR which extracts the text. Then the text tweet content dataset and image extracted text dataset was given as input for preprocessing and feature extraction that uses the NLP. The CNN plays a major role that classifies the tweet contents as positive and negative. Finally, the negative tweets and the user details are collected and sent to the NGO for counseling

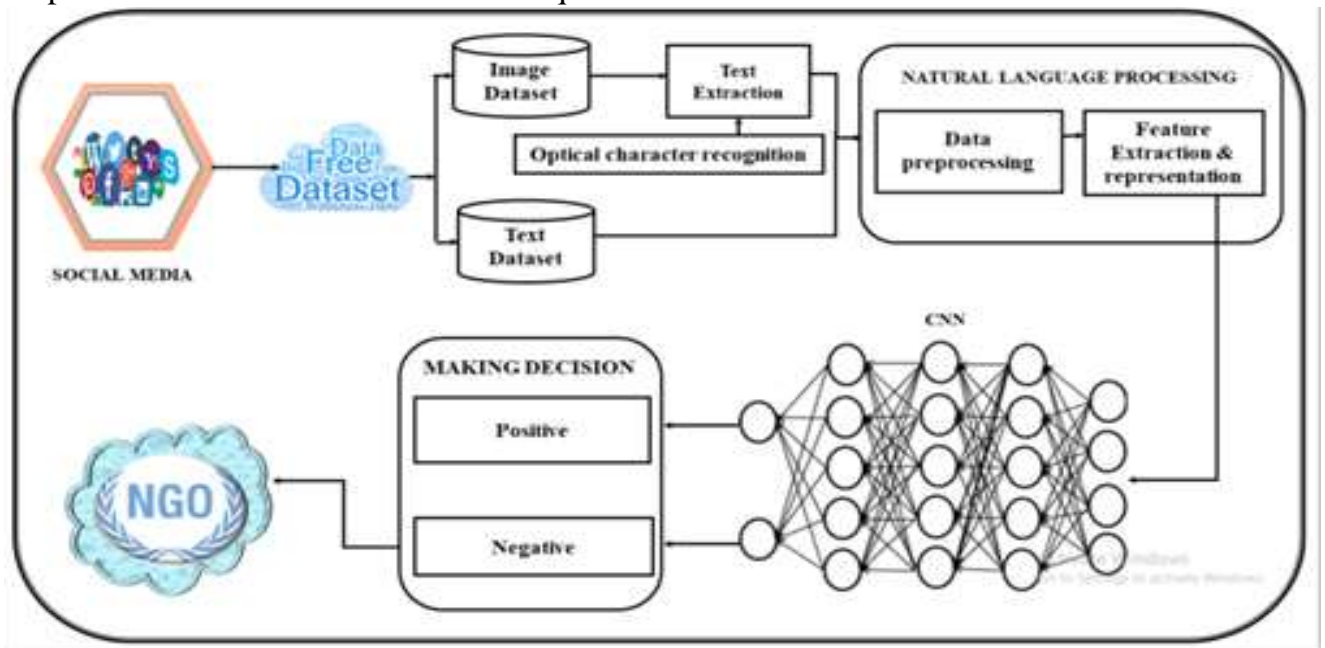

Fig 1. Architecture of psychological stress prediction 
The social media dataset was collected it consists of image data and text_tweet data. The image data consist of images with user_id. The text_tweet data consist of train data and the test data. The training data is labeled as user_id, prediction, and tweet where the user_id is a unique integer identifying the tweet, the prediction is either 1 (positive) or 0 (negative). The test data is labeled as user_id and tweet.

\subsection{Text Recognition from Images}

To express the emotion of the users in the social media, they use to share text, image and video data. Most preferably they use images than video. In this method, the multiple images of the multiple users are fetched from the social media dataset and processed parallelly. The text from the image can be detected and recognized by using Optical Character Recognition (OCR). The OCR is a method to scans the text sequentially in the images to recognize the separate letters from the text line and it can be grouped. The main processes are Preprocessing, Character recognition and Post-preprocessing. The preprocessing is the process of Noise filtering, Image enhancement and character recognition is important the process which includes Pattern recognition or Adaptive recognition in OCR. The post-processing which makes use of co-occurrence frequencies to correct errors. The preprocessing and post-processing are the important process which is used for lexicon driven Optical Character Recognition to detect the text patterns and with high accuracy. By these methods, the text from the multiple images of different users is extracted and can be stored in a CSV file, which consists of two attributes like tweet_id and tweet. Finally, the CSV file is merged with the text tweet content dataset and these are given as an input to the preprocessing.

\subsection{Preprocessing}

The text_tweet dataset is a mixture of URLs, emoticons, symbols, words, and references to people. The text or words and the emoticons are the important keys for the prediction but the other notations like URLs and hashtag can be ignored and it will be removed. The words are also a mixture of extra punctuations, unwanted symbols, and words with many repeated letters and misspelled words. So these unwanted things in the tweet can be removed by preprocessing for standardizing the data. In this paper, Natural language processing is mainly used for preprocessing which consists of some basic preprocessing steps including Structure extraction, Tokenization, Cleaning, Normalization and so on. Here the preprocessing includes converting all the text_tweet to lower case characters, Replacing two or more dots (.) with space and replacing more spaces with a single space. The URLs (hyperlinks) and @ user mentioning can be used by the users while tweeting. The hyperlinks are identified and it can be replaced with the word URL. The user mentioned in the tweet can be replaced as USERMENTIONED word. The emoticon is often used while tweeting so it can be identified and it can be replaced as EMO_POS and EMO_NEG. The hash-tagging was mainly used in social media to quote the trending topics. The hashtag (\#) can be identified and it can be removed. After all these processes individual words in the tweets are processed by removing symbols like (-, '), strip the punctuation from the words, converting the repeated letters in the tweets as regular. Finally validation for the word i.e., checking where the word starts with the alphabet or not.

\subsection{Feature Extraction}

The NLP approaches can be used for feature extraction to build a classifier. The main approaches used for feature extraction in these approaches are N-gram and Word2vec. $\mathrm{N}$-gram is computational linguistics and a sequence of tokens for the n-items from a given sequence of text. Here unigram and bigram methods are used. The frequency distribution of Unigram and Bigram are created and top $\mathrm{N}$-gram is used for the analysis. The single word or tokens in the text for text classification most common and simplest feature. In this process single word is extracted from the train data and frequency distribution was created for each of these words. The top $\mathrm{N}$ words from the frequency distribution used for the dense vector representation. Zipf's law is used for the frequency distribution, it states that the frequency of a word is inversely proportional to its rank in the frequency table for the largest sample of words. For bigram is a pair of words taken from the dataset for classification. This method gives better accuracy in classifying natural languages. After extraction of the $\mathrm{N}$-gram, the features will be represented. For CNN classification dense vector representation is used by assigning the integer index to every word in the data depending upon the rank. The dense vector is the process of assigning the tweet is represented by the vector.

\subsection{Convolutional Neural Network Classification}

Neural network classification which gives more accuracy when compared to the traditional machine learning classifications like Support Vector Machine, Naive Bayes, Maximum entropy etc. Convolutional Neural Networks $(\mathrm{CNN})$ is a combination of deep learning and neural networks which is used for classification to predict result with higher accuracy. It has convolutional layers and pooling operations are performed in these layers. The convolutional layer can interpret the spacial data and it extracts specific features form the data by learning from the number of kernels or filters. By using the two- dimensional sliding window the temporal convolutional operation will be performed in the kernel. To train the CNN dense vector representation is used, here 200 dimension vectors was used to represent the word. The embedding layer is the first layer in matrix form consists of the vocabulary size and the dimension vector for each word. Each word is embedded with the vector and the padding of each vector representation with 0 at the end to the equal of its maximum length. The weight update scheme and binary cross-validation is used for training the data. In this classification, CNN which consist of 4 convolutional layers shown in Figure 2. 


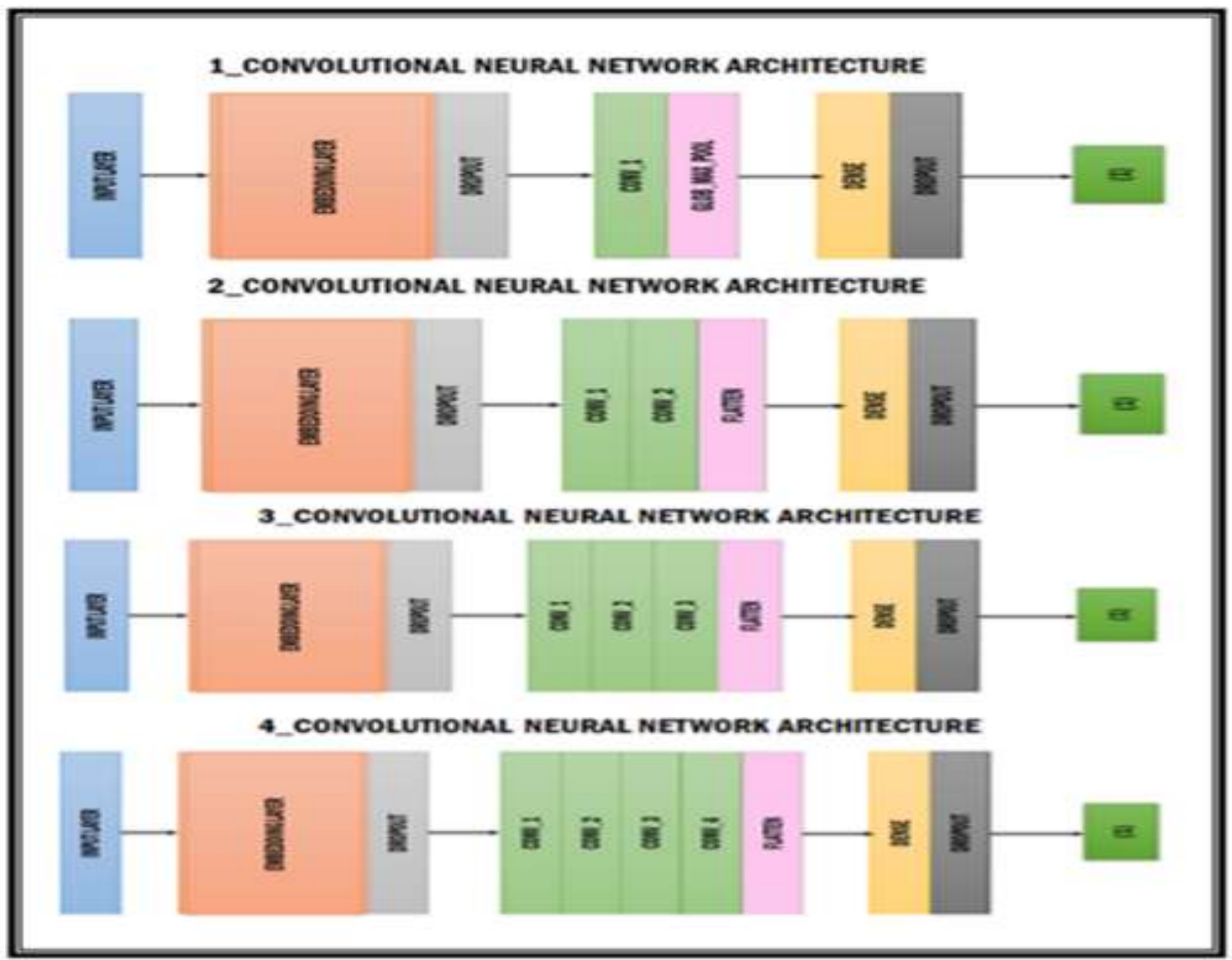

Fig 2. 4-Layered Convolutional Neural Networks for classification

$1-\mathrm{CNN}$ is the first layer which consists of 1-convolution layer and the temporal convolutional operation is performed in this layer with the size of the kernel and the 0-padding. Then the relu activation function is applied to the layer and performs pooling operation. Here the Global maximum pooling is performed to reduce the data dimensionality. The output of the pooling is given as input for the fully connected convolutional layer which produces the single value. With this value, sigmoid activation function is performed which gives probability value as an output. For the network regularization and to avoid over-fitting problem finally the Dropout layer is added at the end of the network.

$$
\begin{aligned}
& \text { relu activation function } \\
& \qquad \begin{array}{c}
f(x)=x^{+}=\max (0, x) \\
\text { sigmoid activation function } \\
f(z)=\frac{1}{1+e^{-z}}
\end{array}
\end{aligned}
$$

\subsection{Connecting to $\mathrm{NGO}$}

After CNN classification of the social media data the predicted result can be labelled as positive (1) which denotes the non- stressed user and negative (0) as stressed user. The details of the stressed users (i.e., user_id) are extracted and it will be given to the NGO for counseling the stressed user. This is one of the methods to identify the stressed user in the social network and by giving counseling to the stressed user it is much possible to reduce the stress level and the suicide rate in the society.

\section{CONCLUSION}

In this paper, we have discussed the machine learning approaches like OCR, NLP and CNN to predict the psychological stress state of the user to reduce the suicidal rate. Here the dataset is collected and pre-processed and trained, then the features are extracted and represented and implemented using Convolutional Neural Networks for the polarity classification. When CNN compared to all other classifiers like Naïve Bayes, Maximum Entropy, SVM, MLP it gives improved and more performance in terms of accuracy shown in Table 1. By using this approach stressed user are predicted earlier and connected to the NGO.

Table I. Comparison of Various Classifiers with Convolutional Neural Network

\begin{tabular}{|l|c|c|}
\hline \multirow{2}{*}{ Classifier } & \multicolumn{2}{c|}{ Accuracy } \\
\cline { 2 - 3 } & Unigram & $\begin{array}{c}\text { Cnigram } \\
\text { Bigram }\end{array}$ \\
\hline Support Vector Machine & 79.5 & 80 \\
\hline Naive Bayes Classifier & 78.16 & 79.68 \\
\hline Multi-Layer Perceptron & 80.1 & 81.04 \\
\hline $\begin{array}{l}\text { Convolutional Neural } \\
\text { Network }\end{array}$ & $\mathbf{8 3}$ & $\mathbf{8 3 . 5 3}$ \\
\hline
\end{tabular}


In future work, we expand the process to include the handling of symbols and extending the emotion range and also including the images handling the colors and face recognition which is more effective to identify the stress level of the user and to reduce the suicidal rate in the society using social media applications.

\section{REFERENCES}

1. Aarushi Agarwal, Prerana Mukherjee, Siddhardh Srivastava, Brejesh Lall, Enhanced Characterness for Text Detection in the Wild, Computer Vision and Pattern Recognition, vol. abs/1712.04927 (2017).

2. Vibhor Goel, Anand Mishra, Karteek Alahari, C.V. Jawahar, Whole is Greater than Sum of Parts: Recognizing Scene Text Words, IEEE International Conference on Document Analysis and Recognition, Washington, DC, USA,pp. 398 - 402 (2013).

3. Kai Wang, Boris Babenko, Serge Belongie, End-to-end scene text recognition, IEEE International Conference on Computer Vision, Barcelona, Spain, pp.1457 - 1464 (2012).

4. Nasukawa, Jeonghee Yi, Sentiment analysis: Capturing favorability using natural language processing, ACM Proceedings of the 2nd international conference on Knowledge capture, Sanibel Island, FL, USA ,pp. 70-77, (2003).

5. M. Johnson Vioulès, B. Moulahi, J. Azé , S. Bringay, Detection of suicide-related posts in Twitter data streams, Vol. 62 , Issue: 1 , (2018).

6. Farman Ali, DaehanKwak, PervezKhan, S.M. RiazulIslam, Kye HyunKim, K.S.Kwak, Fuzzy Ontologybased Sentiment Analysis of Transportation and City Feature Reviews for Safe Traveling, ELSEVIER Emerging Technologies, Vol. 77, pp.33-48, (2017).

7. Yuanbo Gao, Baobin Li, Xuefei Wang, Jingying Wang, Yang Zhou, Shuotian Bai Tingshao Zhu, Detecting Suicide Ideation from Sina Microblog, IEEE International Conference on Systems, Man, and Cybernetics (SMC), (2017).

8. Yuan Zhang, Jie Tang, Jimeng Sun, Yiran Chen, and Jinghai Rao ,Moodcast: Emotion prediction via dynamic continuous factor graph model, IEEE International Conference on Data Mining, (2016).

9. Bridianne O'Dea, Stephen Wan, Philip J.Batterham, Alison L. Calear, Cecile Paris, Helen Christensen, Detecting suicidality on Twitter, ELSEVIER - Internet Interventions, Vol. 2, Issue 2, pp.183-188, (2015).

10. Huijie Lin, Jia Jia, Quan Guo, Yuanyuan Xue, Jie Huang, Lianhong Cai, Ling Feng, Psychological stress detection from cross-media microblog data using deep sparse neural network, IEEE - International Conference on Multimedia and Expo, pp. 1-6, (2014).

11. Anees Ul Hassan, Jamil Hussain, Musarrt Hussain, Muhammad Sadiq, Sungyoung Lee, Sentiment Analysis of Social Networking Sites (SNS) Data using Machine Learning Approach for the Measurement of Depression, IEEE International Conference on Information and Communication Technology Convergence (ICTC), (2017). 